\title{
First Tests of Prototype SCUBA-2 Superconducting Bolometer Array
}

\author{
Adam L. Woodcraft*, Peter A. R. Ade*, Dan Bintley*, Cynthia L. Hunt*, Rashmi V. \\ Sudiwala*, Gene C. Hilton ${ }^{\dagger}$, Kent D. Irwin ${ }^{\dagger}$, Carl D. Reintsema ${ }^{\dagger}$, Michael D. \\ Audley $^{* *}$, Wayne S. Holland** and Mike MacIntosh** \\ *School of Physics and Astronomy, Cardiff University, 5 The Parade, Cardiff, CF24 3 YB UK. \\ ${ }^{\dagger}$ National Institute of Standards and Technology, 325 Broadway, Boulder, CO 80303, USA \\ ${ }^{* *}$ UK Astronomy Technology Centre, Royal Observatory, Blackford Hill, Edinburgh, EH9 3JF, UK
}

\begin{abstract}
We present results of the first tests on a 1280 pixel superconducting bolometer array, a prototype for SCUBA2, a sub-mm camera being built for the James Clerk Maxwell Telescope in Hawaii. The bolometers are TES (transition edge sensor) detectors; these take advantage of the large variation of resistance with temperature through the superconducting transition. To keep the number of wires reasonable, a multiplexed read-out is used. Each pixel is read out through an individual DC SQUID; room temperature electronics switch between rows in the array by biasing the appropriate SQUIDs in turn. Arrays of 100 SQUIDs in series for each column then amplify the output. Unlike previous TES arrays, the multiplexing elements are located beneath each pixel, making large arrays possible, but construction more challenging. The detectors are constructed from $\mathrm{Mo} / \mathrm{Cu}$ bi-layers; this technique enables the transition temperature to be tuned using the proximity effect by choosing the thickness of the normal and superconducting materials. To achieve the required performance, the detectors are operated at a temperature of approximately $120 \mathrm{mK}$. We describe the results of a basic characterisation of the array, demonstrating that it is fully operational, and give the results of signal to noise measurements.
\end{abstract}

To appear in the proceedings of the 24th International Conference on Low Temperature Physics, Orlando, Florida, USA, 10-17th August 2005. (American Institute of Physics, Suite 1N01, 2 Huntingdon Quadrangle, Melville, NY USA.

Keywords: submillimeter, TES, multiplexer, array, bolometer, SQUID, cryogenic

PACS: $07.57 . \mathrm{Kp}, 85.25 . \mathrm{Pb}$

Astronomy at sub-mm wavelengths (hundreds of microns) is relatively new. In the last decade, state of the art systems have improved from single pixel detectors to arrays of hundreds of pixels. There are huge advantages to be gained from increasing the number of pixels still further, if this can be done without compromising performance. This paper describes tests on a prototype 1280 pixel detector sub-array for a new instrument, SCUBA2 [1]. With over 10000 pixels in total, this will give a revolutionary improvement over existing instruments in sensitivity and mapping speed, and bring CCD-style imaging to the sub-mm for the first time.

Bolometers are the most sensitive detectors for broadband measurements at sub-mm wavelengths. Most existing arrays use semiconductors as the temperature sensing element. However, these have reached the sensitivity limits possible for this technology. Moreover, it is difficult to scale such arrays to more than a few hundred pixels since they involve manual steps in the construction of the pixels, and require separate wiring to each detector.

SCUBA-2 uses TES (transition edge sensor) detectors. These obtain high sensitivity from the large change in resistance through the superconducting transition. A constant bias voltage results in negative feedback to temper- ature changes due to changes in incident power, keeping the detector in the superconducting transition without the need for active temperature control. The transition temperature is tuned via the proximity effect by choosing the thicknesses in a molybdenum-copper bi-layer. Thermal isolation of the sensing element is achieved using a silicon nitride membrane [2].

This choice of detector permits an array to be fabricated without assembling pixels individually, and for a multiplexed readout to be used, reducing the wire count to a reasonable level. A similar design is possible using silicon semiconductor detectors, but the noise properties would not meet the requirements of SCUBA-2 [3].

The time division multiplexing system, developed at NIST, has been described elsewhere [4]. Briefly, each pixel is read out through an individual DC SQUID; room temperature electronics switch between rows in the array by biasing the appropriate SQUIDs in turn. Series arrays of 100 SQUIDs for each column then amplify the output. Due to the heat dissipated by these series arrays, they are mounted at a temperature of $1 \mathrm{~K}$. The detectors themselves need to operate at a temperature of approximately $120 \mathrm{mK}$ to achieve the required performance. The SQUID corresponding to each pixel is operated in a flux 


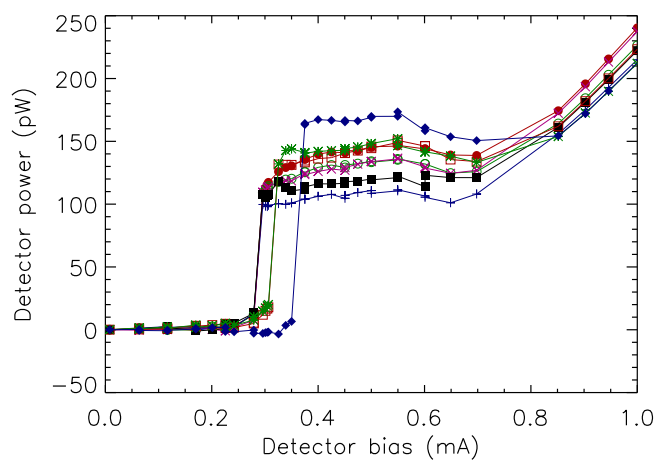

FIGURE 1. Electrical power as a function of bias current for 8 multiplexed pixels (see text).

locked loop; digital electronics [4] are used to select the appropriate feedback setting as each pixel is selected.

In previous implementations, the multiplexer circuits have been located adjacent to the arrays. This is not practical for SCUBA-2, both because of the space which would be required for such a design and because the number of connecting wires would be too large. SCUBA2 therefore uses an in-focal-plane architecture, in which the multiplexing elements are located beneath each pixel. Indium bump bonds carry electrical signals from each pixel to the multiplexer circuitry beneath as well as providing bonding between the multiplexer [4] and detector [2] wafers. The measurements presented here represent the first tests of a detector array using this construction.

The prototype array tests have been carried out in a testbed at Cardiff University. This uses a dilution fridge to reach milliKelvin temperatures. Heated blackbodies viewed through band-defining filters enable the response to sub-mm radiation to be tested.

The flux locked loop for each pixel requires seven parameters to be adjusted appropriately. In full operation, these parameters will be selected automatically. However, for these tests, only manual operation was available. The number of pixels measured at once was thus limited. The largest number of pixels read simultaneously was 40 , with 8 or 16 being typical.

The multiplexer and detectors were found to operate in a stable and reproducible fashion, and operation was not prevented by any interactions between the detectors and the multiplexer despite their proximity. The absolute value and variations in the TES transition temperatures were within specification. Figure 1 shows the dissipated electrical power as a function of current for 8 pixels in a single column read out simultaneously via the multiplexer. The power is constant while the pixels are in the superconducting transition, as is expected for TES sensors. Figure 2 shows the response to modulated sub-

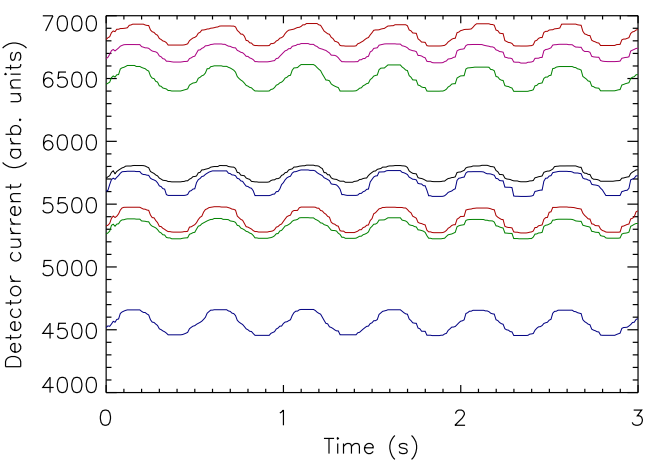

FIGURE 2. Response to modulated illumination for 8 multiplexed pixels (see text).

$\mathrm{mm}$ radiation, again for 8 multiplexed pixels. The offsets are arbitrary, and it should be noted that the modulation height is similar for the different pixels (the scale on the $\mathrm{y}$ axis is arbitrary, but the same for each pixel).

Detector noise is a very important property of the arrays. We have measured the NEP (noise equivalent power) of several pixels by taking noise spectra while modulating a known power from an illuminator at a frequency of $2 \mathrm{~Hz}$. Measurements were taken in the high bias part of the transition as is planned during normal operation. The NEP was then obtained by Fourier transforming the resulting data and comparing the magnitude of the response at the illuminator modulation frequency with the noise floor. A value of $2.5 \times 10^{-17} \mathrm{WHz}^{-1 / 2}$ was measured at a sampling frequency of $50 \mathrm{kHz}$; this is within the specification of $3 \times 10^{-17} \mathrm{WHz}^{-1 / 2}$.

These measurements have therefore demonstrated that the array design functions well, and have permitted the start of construction of the science-grade arrays that will make up the actual instrument. In addition, with an increasing interest in other applications at these wavelengths, there are many potential spin-offs from this technology outside astronomy.

\section{REFERENCES}

1. M. D. Audley, W. S. Holland, W. D. Duncan et al., Nucl. Instrum. Methods Phys. Res. A 520, 479-482 (2004).

2. A. J. Walton, W. Parkes, J. G. Terry et al., IEE Proc.-Sci. Meas. Technol. 151, 110-120 (2004).

3. F. Simoens, P. Agnèse, A. Béguin et al., "Submillimeter bolometers arrays for the PACS/Herschel spectrophotometer," in Millimeter and Submillimeter Detectors for Astronomy II, edited by Jonas Zmuidzinas et al., Proceedings of SPIE 5498, Bellingham, Washington, 2004, pp. 177-185.

4. C. D. Reintsema, J. Beyer, S. W. Nam et al. Rev. Sci. Inst. 74, 4500-4508 (2003). 